\title{
The Effects of High Intensity Neuromuscular Electrical Stimulation on Abdominal Strength and Endurance, Core Strength, Abdominal Girth, and Perceived Body Shape and Satisfaction
}

John Porcari*, Abigail Ryskey, Carl Foster

Department of Exercise and Sport Science, University of Wisconsin-La Crosse, La Crosse, WI USA

Corresponding Author: John Porcari, E-mail: jporcari@uwlax.edu

\section{ARTICLE INFO}

Article history

Received: November 08, 2017

Accepted: January 25, 2018

Published: January 31, 2018

Volume: 6 Issue: 1

Conflicts of interest: None

Funding: This study was funded by a grant from Bio-Medical Research, Ltd, Galway, Ireland

\begin{abstract}
Background: Neuromuscular electrical stimulation (NMES) has been used clinically for many years as a modality to improve muscular strength and endurance. Recently, equipment manufacturers have developed over-the-counter NMES units to target specific muscle groups, particularly the abdominal region. Objective: To study the effects of self-administered neuromuscular electrical stimulation (NMES) on changes in abdominal muscle strength and endurance, core strength, abdominal girth, and subjective measures of body satisfaction and shape. Methods: Fifty-three adults were randomly assigned into high intensity (HI: n=27) or low intensity (LI: $n=26$ ) groups. The NMES device for the LI group had been altered so that subjects felt some tactile sensation, but the intensity was not sufficient to elicit a muscular contraction. All subjects stimulated their abdominal muscles 5 days per week (30 minutes per session) for 6 weeks. Subjects were tested at Baseline, 2, 4, and 6 weeks. Results: The HI group had a significantly greater increase in strength at 4 weeks (19\%) and 6 weeks $(29 \%)$ compared to the LI group and performed significantly more curl-ups than the LI group at 2 weeks (62\%). Both groups had a significant increase in core strength over the course of the study, with no difference between groups. There was no change in abdominal girth between groups. Both groups had significant improvements in body satisfaction from Baseline to 4 weeks and Baseline to 6 weeks, with no significant interaction. Conclusions: Results of the current study indicate that high intensity NMES can significantly increase abdominal strength and endurance compared to LI intensity (control) stimulation. Results for subjective measures tended to favor the HI group, but were less conclusive, since the LI group also had some positive changes.
\end{abstract}

Key words: Prone Plank Test, Curl-ups, Slendertone, Placebo

\section{INTRODUCTION}

Neuromuscular electrical stimulation (NMES) is a well-established therapeutic modality that has been used for many years in the practice of physical therapy. When traditional exercise is not possible due to injury or surgery, NMES may be used as a means of maintaining muscular strength and minimizing atrophy due to immobilization (Hainaut \& Duchateau, 1992). In the 1960's, Kots (1977) reported using NMES as a training adjunct with elite athletes in the former Soviet Union and reported strength improvement of $30-40 \%$. He suggested that NMES might be more effective than volitional exercise for strength improvement. In recent years, fitness equipment companies have marketed NMES devices for healthy individuals as an alternative way to improve muscle strength and endurance and improve body composition. These devices are designed for many different muscle groups, but are of particular interest for the abdominal region. The desire of Americans to have a trim waist and flat stomach without having to exercise is an attractive option for many people. There are conflicting results regarding the effects of NMES on the abdominal musculature. Many studies have demonstrated significant improvements in abdominal strength and endurance, perceived muscle tone, and body satisfaction following NMES training (Alon et al., 1987; Alon et al., 1992, Abendroth-Smith \& Sword, 1977; Anderson et al., 2006, Ballantyne \& Donne, 1999; Porcari et al., 2005), while others have shown no improvement in these parameters (Aikman, et al., 1985; Porcari et al., 2002).

Whether or not improvements in muscular strength and endurance are seen with NMES is reasonably dependent upon the strength of the resulting contraction. Those studies that have utilized contractions in excess of $60 \%$ of maximum voluntary contraction (MVC) have shown positive benefits (Currier \& Mann, 1983; Muffiuletti, 2002; Selkowitz, 1989; and Soo et al., 1988). Many over-thecounter NMES devices do not deliver a strong enough 
stimulus to reach this threshold. A previous study in our laboratory (Porcari et al., 2002) investigated the effects of training with one of these devices (Body Shapers, International, Model BM1012BI) on the strength of various muscle groups, body composition, and physical appearance. No statistically significant improvements in any of the outcome measurements were found. The conclusion of the study was that the stimulator and electrodes used to deliver the stimulation were poorly constructed and did not deliver a strong enough current to elicit a contraction of sufficient strength to induce gains in strength. Additionally, the stimulation was very uncomfortable for subjects. Measures taken during stimulated contractions suggested that the percentage of maximal strength that the muscles were contracting was less than $20 \%$ of MVC.

In an attempt to overcome this deficiently and elicit stronger muscular contractions, Bio-Medical Research, Ltd. (Galway, Ireland) developed an NMES belt that targets the abdominal muscles. The device delivers an electrical current to the abdominal region using medical-grade adhesive pads placed over motor units of the abdominal musculature. The belt is cleared by the Food and Drug Administration (FDA) in the United Sates to increase the strength and tone of the abdominal muscles. A subsequent study from our laboratory (Porcari et al., 2005) supported these claims, as abdominal strength improved $49 \%$ and abdominal endurance increased $72 \%$ compared to a non-stimulation control group. There was also an increase in perceived muscle tone in all subjects in the stimulation group, as well as reduction in abdominal girth. A criticism of the above study was that the control group did not receive any intervention. Thus, it was felt that some of the improvement in the stimulation group, particularly regarding subjective outcome measures, was attributable to the placebo effect. Bio-Medical Research, Ltd. has developed a newer NMES device called the Slendertone ${ }^{\circledR}$ System Abs belt, which has the same indications for use as the Slendertone ${ }^{\circledR}$ FLEX, but with higher intensity levels. The higher intensity levels are purported to elicit stronger, more effective muscle contractions. The purpose of this study was to determine the efficacy of the Slendertone ${ }^{\circledR}$ System Abs belt for increasing abdominal muscular strength and endurance, improving core muscle strength, decreasing abdominal girth, and improving self-perceived body satisfaction and abdominal muscle tone in healthy, middle-aged adults.

\section{METHODS}

\section{Subjects}

Fifty-six adult volunteers from the La Crosse, Wisconsin area were recruited through an advertisement in the local newspaper. Inclusion criteria required the subjects to be between 25 and 55 years old, to be healthy by their own report, to have a body mass index (BMI) between 18 and 30, and not to have been involved in any type of formal abdominal training program within the previous 6 months. In addition, subjects with any implanted medical devices (pacemaker, pump, catheter, etc.), insertion or removal of an IUD contraceptive device (i.e. coil) within the previous month, or who were currently pregnant or had given birth in the previous three months were excluded. Subjects were randomly assigned into one of two groups: a high intensity treatment group (HI) or a low intensity treatment group (LI). Group assignment was randomized and training was double-blinded. Both groups were instructed not to alter their diet or engage in any additional exercise over the course of the 6-week study period. All subjects gave written informed consent prior to participating in the study and the protocol was approved by the Institutional Review Board for the Protection of Human Subjects at the University of Wisconsin-La Crosse. Subjects in both groups received a $\$ 200$ honorarium and a stimulation belt at the conclusion of the study.

\section{Testing}

All subjects completed an identical battery of tests at Baseline and at 2, 4, and 6 weeks of the study protocol. Testing included a series of questionnaires, anthropometric measurements, and determination of abdominal muscle strength, abdominal endurance, and core muscle strength. All tests were given in the same order for all subjects. Testers were blinded to group assignment of the subjects.

\section{Questionnaires}

Subjects completed two questionnaires: The Body Satisfaction Scale and an Overall Results Questionnaire. The Body Satisfaction Scale has been used in a previous study (Porcari et al., 2005) and assessed responses to 10 opposite descrip-tors of body satisfaction. Each set of descriptors was given a score between one (most negative) and five (most positive). At the final visit, participants in both groups also complet-ed an Overall Results Questionnaire. The questionnaire was a simple 13-item questionnaire that asked for a subject's agreement or disagreement with a set of statements regard-ing their perceived benefits from participating in the study.

\section{Anthropometric Measures}

Body weight was measured to the nearest 0.1 kilogram using a standard laboratory beam scale and height was measured to the nearest 0.1 centimeter using a stadiometer. Body mass index (BMI) was calculated from height and weight. Two circumference measurements were taken. One measurement (abdominal measurement) was taken horizontally at the level of the natural waist (the smallest circumference between the ribs and the iliac crest). The second measurement (waist measurement) was taken at the level of the umbilicus. Two measurements were taken at each site. A third measurement was taken if there was greater than one centimeter difference between the first two measurements. The average of the two closest measurements was used in the analysis. All abdominal and waist circumference measurements were made by the same examiner throughout the study using a spring-loaded tape measure. 


\section{Abdominal Strength}

Abdominal strength was measured by having the subject perform five isometric contractions using an isokinetic dynamometer (Biodex, USA). The subject rested supine on a movable bench in a bent-knee position. The lever arm of the isokinetic dynamometer was set horizontal with the ground (180 degrees) and the padded extension arm was placed just below the nipple line on the lower third of the sternum. The height of the bench was adjusted for each subject so that the extension arm remained at 180 degrees. Each subject was given several practice trials to make sure the position of the lever arm was comfortable on their chest. Subjects then performed five maximal isometric contractions, with $30 \mathrm{sec}-$ onds rest between repetitions. The average torque measurement of the highest two repetitions was used in the analysis. Reliability of the abdominal strength test in our laboratory is $\mathrm{ICC}=.96$.

\section{Abdominal Endurance}

Abdominal endurance was assessed using the American College of Sports Medicine (ACSM) paced curl-up test (ACSM, 2000). The subjects were in a supine position on a mat with the knees bent to 90 degrees (measured with a goniometer). The subject's arms were at the side, palms facing down, with the middle fingers touching a piece of tape. A second piece of tape was placed $8 \mathrm{~cm}$ (for those who were $\geq 45$ years) or $12 \mathrm{~cm}$ (for those who were $<45$ years) from the first piece of tape. The subject's shoes remained on during the test. The individual completed slow, controlled curl-ups to lift the shoulder blades off the mat (trunk makes a 30-degree angle with the mat) in time with a pre-recorded tape at a pace of 40 curl-ups per minute. The subject performed as many curl-ups as possible without pausing. The test was terminated when the subject could no longer keep up with the pace of the tape or their fingers could not reach the second piece of tape. The reliability of the abdominal endurance test in our laboratory is $\mathrm{ICC}=.83$.

\section{Core Strength}

Core strength was measured using the Prone Plank test (Quinn, 2008). Subjects assumed the prone "plank" position, with their full body weight supported only by the forearms and toes. Their body was straight with the elbows parallel to each other and directly under the shoulders. They held this "plank" position for a period of 60 seconds. At the end of the 60 second period, the subjects successively raised each limb individually for a period of 15 seconds each. They then were to raise their right arm and left leg for 15 seconds then their left arm and right leg for 15 seconds. Upon completion of the limb movements, the subjects returned to the plank position for 30 seconds. These series of movements were continued until the subject could no longer continue or was no longer able to maintain a straight body position. The total hold time was measured for each subject. The reliability of the prone plank test in our laboratory is $\mathrm{ICC}=.90$.

\section{Training}

All subjects underwent stimulation five times per week for six weeks. Each session was 30 minutes in duration. The NMES device used in the current study was the Slendertone ${ }^{\circledR}$ System Abs belt (Bio-Medical Research, Ltd. (Galway, Ireland). The belt uses three pre-gelled electrodes to deliver an electrical current to the abdominal region. The HI group used the stimulation belt that is currently on the market. The LI group used the same belt, but the stimulator had been altered so that the electrical current was strong enough to cause some tactile sensation, but was not strong enough to elicit a visible muscular contraction. Both groups used program number 3 on the stimulation controller. Each subject was given an individual orientation session, during which they were supervised for their first stimulation session. All other stimulation sessions were completed on their own. Subjects in both groups were encouraged to use the highest tolerable level on their stimulator to achieve the strongest possible contractions. Subjects recorded the maximum intensity reached during each stimulation session in a training log.

\section{Statistics}

Standard descriptive statistics were used to characterize the subject population. Changes in anthropometric data, the Body Satisfaction Scale, abdominal strength, abdominal endurance, and core strength were analyzed using a 3-way ANOVA with repeated measures (Group X Gender X Time). Since there were no differences in the responses of male and females, data were collapsed across gender. Data were then analyzed with a 2-way ANOVA with repeated measures (Group X Time). When there was a significant $\mathrm{F}$ ratio, Tukey's post-hoc tests were used to isolate pairwise differences. A Mann Whitney-U test was used to compare differences in the frequency of responses between groups for the Shape Evaluation Scale over the four testing periods. Alpha was set at 0.05 to achieve statistical significance for all analyses. Data were analyzed using SPSS version 25.0.

\section{RESULTS}

Fifty-three of the original 56 subjects successfully completed the study. One member of the LI group withdrew from the study due to dissatisfaction with the training intensity delivered by the attenuated device and another subject in the LI group was disqualified for doing additional sit-ups during the study period. One subject in the HI group was withdrawn from the study after experiencing heavier than usual blood flow during menses. For the final analysis there were 27 subjects in the HI group (14 male and 13 female) and 26 subjects in the LI group (12 male and 14 female). Descriptive characteristics of the subjects who completed the study are presented in Table 1. There were no significant differences between groups at the start of the study for any of the outcome measures.

\section{Anthropometric Measurements}

Results for the anthropometric data collected during the study are presented in Table 2 . There were no significant 
Table 1. Descriptive characteristics of the subjects who completed the study.

\begin{tabular}{|c|c|c|c|c|}
\hline Group & Age (yr) & Height (cm) & Weight (kg) & BMI \\
\hline \multicolumn{5}{|c|}{ High Intensity } \\
\hline Males & $39.7 \pm 8.0$ & $178.3 \pm 6.4$ & $79.8 \pm 7.7$ & $25.2 \pm 2.4$ \\
\hline Females & $39.5 \pm 9.8$ & $161.9 \pm 5.6$ & $62.1 \pm 5.5$ & $23.7 \pm 1.9$ \\
\hline Overall & $39.6 \pm 8.7$ & $170.4 \pm 10.2$ & $71.4 \pm 11.4$ & $24.4 \pm 2.2$ \\
\hline \multicolumn{5}{|c|}{ Low Intensity } \\
\hline Males & $37.3 \pm 9.0$ & $178.0 \pm 8.1$ & $82.7 \pm 8.6$ & $26.2 \pm 2.6$ \\
\hline Females & $40.7 \pm 8.2$ & $168.7 \pm 6.6$ & $72.3 \pm 7.3$ & $25.4 \pm 2.3$ \\
\hline Overall & $38.8 \pm 8.5$ & $173.0 \pm 8.6$ & $77.2 \pm 9.4$ & $25.7 \pm 2.5$ \\
\hline
\end{tabular}

Values represent mean and standard deviation

Table 2. Changes in body weight, BMI, waist circumference, and abdominal circumference over the course of the study.

\begin{tabular}{llcccc}
\hline Variable & Group & Baseline & Week 2 & Week 4 & Week 6 \\
\hline Body Weight (kg) & High Intensity & $71.4 \pm 11.4$ & $71.5 \pm 11.4$ & $71.5 \pm 11.4$ & $71.7 \pm 11.4$ \\
& Low Intensity & $77.2 \pm 9.4$ & $77.3 \pm 9.5$ & $77.0 \pm 9.7$ & $77.2 \pm 9.7$ \\
Body Mass Index & High Intensity & $24.4 \pm 2.2$ & $24.4 \pm 2.3$ & $24.4 \pm 2.3$ & $25.4 \pm 2.4$ \\
& Low Intensity & $25.7 \pm 2.5$ & $25.8 \pm 2.4$ & $25.7 \pm 2.5$ & $25.7 \pm 2.5$ \\
Waist Circumference (cm) & High Intensity & $82.3 \pm 9.2$ & $81.9 \pm 9.6$ & $80.4 \pm 13.7$ & $82.0 \pm 9.5$ \\
& Low Intensity & $85.0 \pm 8.6$ & $84.6 \pm 8.7$ & $83.0 \pm 8.8$ & $84.3 \pm 8.9$ \\
Abdominal Circumference (cm) & High Intensity & $90.0 \pm 7.9$ & $88.4 \pm 8.3$ & $88.0 \pm 8.6$ & $87.6 \pm 8.3$ \\
& Low Intensity & $94.0 \pm 7.5$ & $92.4 \pm 7.3$ & $90.5 \pm 7.4$ & $90.5 \pm 7.4$ \\
\hline
\end{tabular}

Values represent mean and standard deviation

changes in body weight, BMI, abdominal circumference, or waist circumference in either group over the course of the study.

\section{Abdominal Strength}

Abdominal strength for each group at each time point are presented in Figure 1. There was a significant main effect across testing time $(\mathrm{p}<.001)$ and a significant interaction between groups across time $(\mathrm{p}<.001)$. There were no significant changes for the LI group across the 6-week study. The HI group had significant increases in strength at weeks 4 and 6 compared to Baseline. Changes between groups were statistically significant at weeks 4 and 6 .

\section{Abdominal Endurance}

Curl-up performance of the two groups is presented in Figure 2. There was a significant main effect across testing time $(\mathrm{p}<.001)$ and a significant interaction between groups across time $(p=.034)$. There were no significant increases for the LI group at any time point compared to Baseline. The HI group had significant improvements in curl-up performance after 2, 4, and 6 weeks compared to Baseline. Changes between groups were statistically significant only at week 2 .

\section{Prone Plank Test}

Changes in prone plank test performance are presented in Figure 3. There was a significant main effect across time $(\mathrm{p}=.001)$, but there was no significant interaction between groups across time $(p=.27)$. There were no significant in-

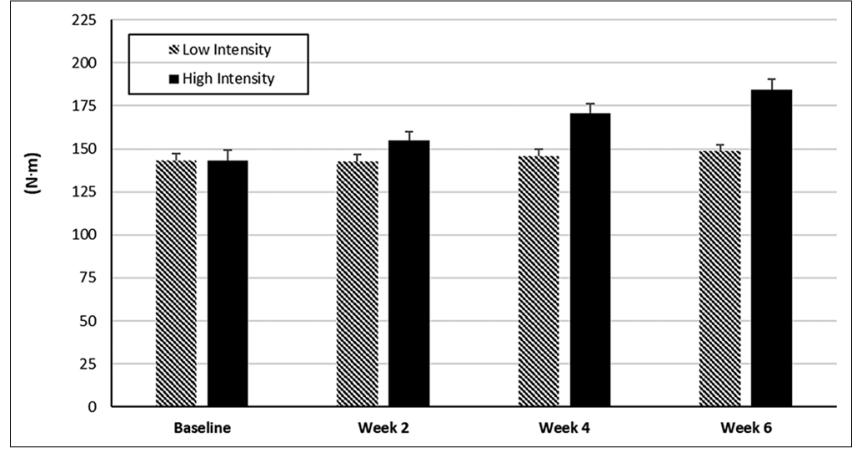

Figure 1. Changes in abdominal strength over the course of the 6-week study. Values represent mean and standard deviation

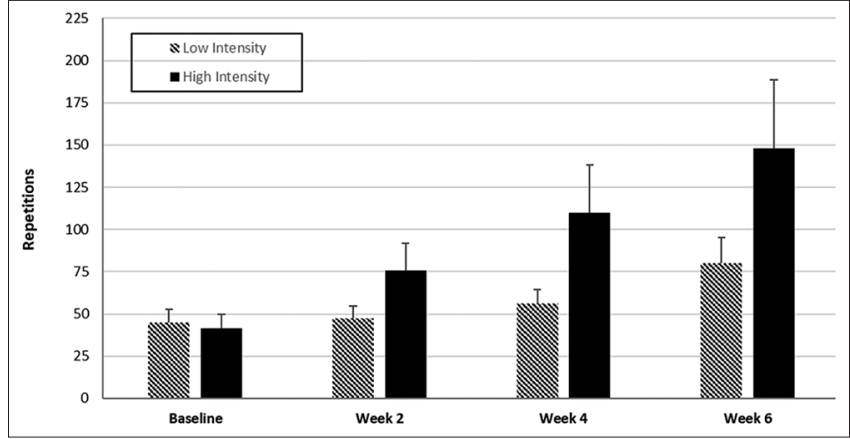

Figure 2. Changes in curl-ups completed over the course of the 6-week study. Values represent mean and standard deviation

creases for the LI group at any time point compared to Baseline. The HI group had significant improvements in prone plank performance at 4 and 6 weeks compared to Baseline, 
but these differences were not significantly different than the LI group at the same time points.

\section{Questionnaire Data}

Summary data for the Body Satisfaction Scale are presented in Table 3. There was a significant main effect across time $(p<.001)$, but there was no significant interaction between groups and time $(\mathrm{p}=.43)$. Both groups had significant improvements in the total score at 4 weeks and 6 weeks compared to Baseline.

For the Overall Results Questionnaire, significantly more subjects in the HI group reported that they noticed positive results after using the belt compared to the LI group (96\% vs. $56 \%$ ). Significantly more subjects in the HI group also reported an improvement in the perceived firmness $(92 \%$ vs. $63 \%)$ and tone ( $81 \%$ vs. $56 \%$ ) of their stomach muscles compared to the LI group. An improvement in perceived strength of the abdominal muscles was reported by $92 \%$ of the HI group compared to $70 \%$ of the LI group. However, this difference was not statistically significant. Significantly more subjects in the HI group felt that using the belt was as effective as sit-ups compared to the LI group ( $81 \%$ vs. $44 \%$ ).

\section{DISCUSSION}

The present study found $19 \%$ and $29 \%$ gains in abdominal strength after 4 and 6 weeks of high intensity stimulation, respectively. These results are in line with results from other studies that have used EMS to stimulate the abdominal musculature (Alon et al., 1987; Alon et al., 1992; Ballantyne \& Donne, 1999). The results of Alon et al. (1987) and Alon et al. (1992) are virtually identical to those of the current study, as they found increase of $20.8 \%$ and $19.6 \%$, respectively, after 4 weeks of EMS training. Since Alon's studies were both 4 weeks in duration, comparisons beyond that point are not

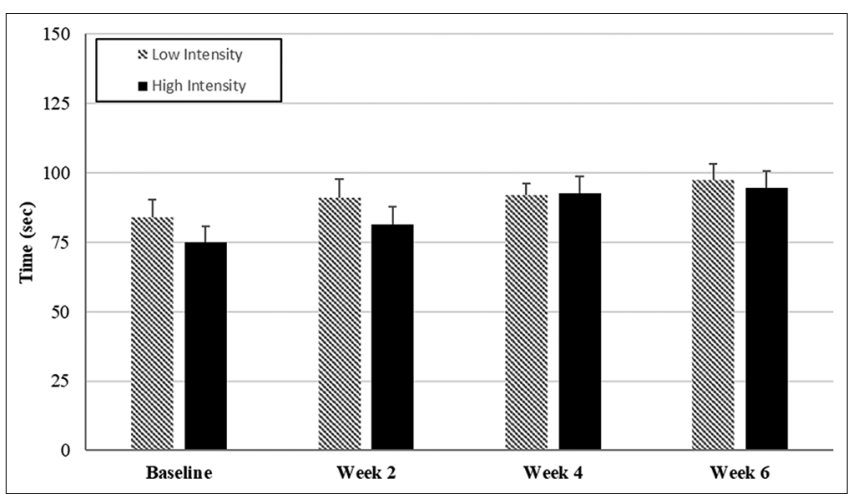

Figure 3. Changes in prone plank time over the course of the 6-week study. Values represent mean and standard deviation possible. The magnitude of the strength improvements seen in the current study are less than those reported previously in our laboratory using a previous version of the belt (Porcari et al., 2005). In the current study a $19 \%$ improvement was apparent after 4 weeks, compared to a $34 \%$ strength increase in the earlier study after the same amount of time. In the previous study strength improvement was not measured at 6 weeks; however, using linear extrapolation, improvement would have been approximately $46 \%$, compared to $29 \%$ in the current study. It has been suggested that in the earlier study a greater number of training sessions were supervised, which may have led to increased compliance with the treatment protocol; hence greater strength improvement. However, the device used in the current study recorded the frequency of stimulation sessions as well as the peak stimulation level used during each session. Attendance to the number of training sessions for every individual in the study was exactly as prescribed ( 5 sessions per week for 6 weeks). Peak intensity was slightly lower in the current study versus the earlier study. However, since the available intensity of the stimulator used in the current study (Slendertone ${ }^{\circledR}$ System Abs) was stronger than that used in the previous study (Slendertone ${ }^{\circledR}$ FLEX), the intensity of the achieved contractions were assumed to be similar.

The HI group had significantly greater improvements in abdominal endurance at 2 weeks compared to the LI group. The data at weeks 4 and 6 also indicated a strong trend in favor of the HI group. However, due to the large standard deviation of scores, the difference was not statistically significant at either time point. The percentage increases in the HI group at 2, 4, and 6 weeks were $64 \%, 133 \%$, and $243 \%$, respectively, compared to $2 \%, 15 \%$, and $73 \%$ for the LI group at the same time points. The difference in improvement between the HI and LI groups were $62 \%, 118 \%$, and $170 \%$ at weeks 2,4 , and 6 . The magnitude of the net improvement for the HI group in the current study was larger than previously found in our laboratory (Porcari et al., 2005). When looking at the raw data, it was observed that two male subjects in the HI group did 575 and 600 curl-ups at the 4-week time period. These same individuals did 800 and 797 curl-ups, respectively, at the 6-week testing mark. As the curl-ups for these individuals were conducted according to protocol, their data was included in the final analysis. Had the data for these two individuals been removed from the data set, the net improvement between the HI and LI groups at weeks 4 (the only comparable testing time between the two studies), would have been $40 \%$ in favor of the HI group. This is comparable to the $49 \%$ improvement seen in the previous study at the same time point.

Another fact to point out was that the LI group had a $15 \%$ improvement in curl-up performance at the 4 - Week

Table 3. Total score on the Body Satisfaction Scale for both groups over the course of the study

\begin{tabular}{lcccc}
\hline Group & Baseline & Week 2 & Week 4 & Week 6 \\
\hline High Intensity & $25.1 \pm 6.44$ & $27.8 \pm 5.36$ & $29.4 \pm 5.09 *$ & $31.4 \pm 5.11^{*}$ \\
Low Intensity & $24.3 \pm 5.86$ & $26.5 \pm 5.05$ & $28.9 \pm 5.46^{*}$ & $30.4 \pm 4.44^{*}$ \\
\hline
\end{tabular}

Values represent mean and standard deviation.* Significantly different than Baseline $(p<.05)$. 
testing time and a $73 \%$ improvement at the 6 - Week testing point. In the previous study by Porcari et al. (2005), a passive control group (no stimulation) had a $28 \%$ improvement in curl-up performance after 8 weeks. This would indicate that there is learning effect associated with performance of the curl-up test. However, the magnitude of the improvement over time, coupled with the fact that the subjects in the current study were receiving some simulation, even though it was at a very low level, suggests that the LI group may have had some improvement in muscular performance. Consistent with this finding is the report of Alon et al. (1997) of a 14\% increase in strength when subjects received very low levels of abdominal electrical stimulation (just enough to elicit a visible tetanic contraction) for 3 hours per day. Another possibility is that because tests like the curl-up test are effort dependent; improvements may have reflected an increased effort on the part of subjects. If they felt that the belt was providing a benefit, they may have tried harder during the later testing sessions.

Results for core strength testing showed significant improvements for both groups. The HI group improved 18\% after 6 weeks and the LI group improved $12 \%$ over the same time frame. Since the prone plank test is a novel test for most people, performance on the test depends a great deal on technique and balance. It is plausible that the overall improvement reflected an improvement in technique, which may have masked any therapeutic difference that may have existed between the groups.

There were no significant changes in any of the anthropometric measures over the course of the study. There was a non-significant decrease in abdominal circumference (the smallest circumference between the ribs and the iliac crest) in both groups. This finding is in conflict with the results of a previous study completed in our laboratory (Porcari et al., 2005). That study found a decrease of 2.6 centimeters in abdominal circumferences after 8 weeks of stimulation. The magnitude of the decrease in abdominal circumference in the above study is similar to that found in the current study (2.4 cm for the HI group and $3.5 \mathrm{~cm}$ for the LI group). However, because there were changes in both groups and because there were no changes in body weight in either group, the decreases were attributed to a consistent evaluator error. Abendroth-Smith and Sword (1977) also failed to find reductions in abdominal girth following 8 weeks of NMES, despite significant improvements in strength.

Several studies have found abdominal NMES training to have a positive effect on self-perception and body image measures (Anderson et al., 2006; Porcari et al., 2005). This study also found significant improvements in the subjective data. However, positive improvements were seen in both groups. Results of the Body Satisfaction Scale were virtually identical between groups, with both the HI and LI groups improving their scores after 4 and 6 weeks of the study. Additionally, even though the HI group had subjectively greater improvements in perceived abdominal firmness and tone than the LI group, the LI group also perceived some benefit. There are a number of possible explanations as to why the LI group had improvements in the subjective tests. In the studies by Porcari et al. (2005) and Anderson et al. (2006), subjects in the control group did not receive any intervention. In the current study, the LI group (designed to be the control group) used belts that elicited some tactile sensation, but no visible muscular contraction. It is likely that because the LI group did feel something, they felt like they were getting a benefit. Anderson et al. (2006) attributed improvements in subjective measures to improvements in abdominal and waist circumference. This could have also played a role in the current study. Even though subjects were not told their previous scores at follow-up testing sessions, many of them could remember their previous values. Thus, since some of knew that they had completed more sit-ups or knew that their abdominal circumference was less during the earlier testing sessions; they assumed that the belt was working. Thus, it would be logical for them to feel better about their midsection.

It was also likely that many of the subjects had seen advertising about the positive results from the previous study (Porcari et al., 2005). That study found significant improvements in muscle strength and endurance and $100 \%$ of the subjects felt that the NMES device improved their abdominal firmness and tone. Thus, subjects probably entered the current study expecting positive benefits. Because none of the subjects had used electrical stimulation previously, they did not know what NMES was supposed to feel like. Even though subjects in the LI group were receiving a low threshold stimulus, they were fooled into thinking that it was working. It was interesting to note that $81 \%$ of the HI group and $44 \%$ of the LI group felt that using the belt was as effective as sit-ups. This further illustrates the magnitude of the placebo effect, since the LI device did not elicit any visible muscle contraction.

The strength of the current study was that the control group actually underwent some sort of intervention, as opposed to just going through all of the testing at the various time points. Additional strengths of the study were that the examiners were blinded to group assignment and the study was overseen by an external study monitor. All facets of data collection and data input were independently verified prior to analysis. The major limitation of the study was that training sessions were carried out independently by subjects and were not monitored. We did not feel monitoring was necessary, since the simulator recorded the number of sessions completed, average intensity of stimulation, and peak intensity. Despite this, future studies may want to include training supervision. Also, since the simulation belt provides an improvement in muscular strength and endurance without having to do traditional abdominal exercises, a similar study could be conducted in individuals in which abdominal exercises may be contraindicated (e.g. people with low back pain).

\section{CONCLUSIONS}

High intensity electrical muscle stimulation to the abdominal musculature resulted in greater improvements in abdominal muscular strength and endurance compared to low intensi- 
ty (control) stimulation. The non-significant improvements in abdominal endurance and core strength in the LI group were thought to be due to a learning effect associated with the tests performed, the placebo effect, or a combination of both factors. It is also possible that these results could have been due to the fact that the LI group was receiving a very low level of electrical stimulation, which could have caused some positive neuromuscular changes. Since the results of the current study and data available in the literature present conflicting results regarding the effect of NMES on body composition and perceived body image, additional well-designed studies are needed to clarify this issue. Additionally, future studies may want to compare the potential benefits of traditional abdominal exercises vs. NMES applied to the abdominal musculature.

\section{REFERENCES}

Abendroth-Smith, J., \& Sword, K. (1977). Toning and strengthening predominant muscle groups through electrical muscle stimulation. Medicine and Science in Sports and Exercise, 29(5), 165.

Aikman, H.D., Majerus, J.J., Van Wart, K.W., Barr, J.O., \& Cook, T.M. (1985). Effects of electrical stimulation vs. sit-ups on abdominal strength and endurance. Physical Therapy, 65, 596.

Alon, G., Frederickson, R., Gallagher, L., Rehwoldt, C.T., Guillen, M., Putnam-Pement, M.L., \& Barhart, J.B. (1992). Electrical stimulation of the abdominals: The effects of three versus five weekly treatments. Journal of Clinical Electrophysiology, 4, 5-11.

Alon, G., McCombe, S.A., Koutsantinis, S., Stumphauzer, L.J., Burgwin., K.C., Parent, M.M., \& Bosworth, A. (1987). Comparison of the effects of electrical stimulation and exercise on abdominal musculature. The Journal of Orthopaedic and Sports Physical Therapy, 8, 567-573.

Alon, G., \& Taylor, D.J. (1997). Electrically elicited minimal visible tetanic contraction and its effect on abdominal muscles strength and endurance. European Journal of Physical Medicine and Rehabilitation, 7, 2-6.

American College of Sports Medicine. (2000). ACSM's Guidelines for Exercise Testing and Prescription. $6^{\text {th }}$ Edition. Lippincott Williams \& Wilkins, Philadelphia.
Anderson, A.G., Murphy, M.H., Murtagh, E., \& Nevill, A. (2006). An 8-week randomized controlled trial on the effects of brisk walking, and brisk walking with abdominal electrical stimulation on anthropometric, body composition, and self-perception measures in sedentary adult women. Psychology of Sport and Exercise, 7, 437-451.

Ballantyne, E., \& Donne, B. (1999). Effects of neuromuscular electrical stimulation on static and dynamic abdominal strength and endurance in healthy males. Sports Science, 431.issue and page number

Currier, D.P., \& Mann, R. (1983). Muscular strength development by electrical stimulation in healthy individuals. Physical Therapy, 63, 915-921.

Hainaut, K., \& Duchateau, J. (1992). Neuromuscular electrical stimulation and voluntary exercise. Sports Medicine, 14(2), 100-113.

Kots, Y. (1977). Electrostimulation of skeletal muscles. $\mathrm{Ca}$ nadian-Soviet Exchange Symposium, Concordia University.

Maffiuletti, N.A., Dugnani, S., Folz, M., Di Pierno, E., \& Mauro, F. (2002). Effect of combined electrostimlation and plyometric training on vertical jump height. Medicine and Science in Sports and Exercise, 34, 1638-1644.

Mueller, E.A. (1959) Training muscle strength. Ergonomics, 2, 216-222.

Porcari, J.P., Palmer McLean, K., Foster, C., Kernozek, T., Crenshaw, B., \& Swenson, C. (2002). Effects of electrical muscle stimulation on body composition, muscle strength, and physical appearance. Journal of Strength and Conditioning Association Research, 16, 165-172.

Porcari, J.P., Miller, J., K. Cornwell., Foster, C., Gibson, M., McLean, K., \& Kernozek, T. (2005). The effects of neuromuscular electrical stimulation training on abdominal strength, endurance, and selected anthropometric measures. Journal of Sports Medicine and Science, 4, 66-75.

Quinn, E. (2008) Core muscle strength and stability test. [Online] Available: http://sportsmedicine.about.com/od/ bestabexercises/a/core_test.htm(October 23, 2007)

Selkowitz, D.M. (1989). High frequency electrical stimulation in muscle strengthening. American Journal of Sports Medicine, 17, 103-111.

Soo, C.L., Currier, D.P., \& Threlkeld, A.J. (1988). Augmenting voluntary torque of healthy muscle by optimization of electrical stimulation. Physical Therapy, 68, 333-337. 